\title{
Space-based geoengineering: challenges and requirements
}

\author{
C R McInnes \\ Department of Mechanical Engineering, University of Strathclyde, Glasgow G1 1XJ, UK. \\ email: colin.mcinnes@strath.ac.uk
}

The manuscript was received on 11 November 2008 and was accepted after revision for publication on 15 September 2009.

DOI: 10.1243/09544062JMES1439

\begin{abstract}
The prospect of engineering the Earth's climate (geoengineering) raises a multitude of issues associated with climatology, engineering on macroscopic scales, and indeed the ethics of such ventures. Depending on personal views, such large-scale engineering is either an obvious necessity for the deep future, or yet another example of human conceit. In this article a simple climate model will be used to estimate requirements for engineering the Earth's climate, principally using space-based geoengineering. Active cooling of the climate to mitigate anthropogenic climate change due to a doubling of the carbon dioxide concentration in the Earth's atmosphere is considered. This representative scenario will allow the scale of the engineering challenge to be determined. It will be argued that simple occulting discs at the interior Lagrange point may represent a less complex solution than concepts for highly engineered refracting discs proposed recently. While engineering on macroscopic scales can appear formidable, emerging capabilities may allow such ventures to be seriously considered in the long term. This article is not an exhaustive review of geoengineering, but aims to provide a foretaste of the future opportunities, challenges, and requirements for space-based geoengineering ventures.
\end{abstract}

Keywords: macroengineering, geoengineering, climate change

\section{INTRODUCTION}

Human civilization has developed during a time when the climate has been in a relatively benign state, with modern civilization quickly developing during a temperate period between extremes. These favourable circumstances have allowed rapid global population growth combined with relative affluence in the West. However, the recent controversy surrounding humandriven climate change has brought into sharp focus the fact that the climate is not static. The popular view of the climate as being perpetually in equilibrium is only due to the narrow window of human history through which we view the past.

Natural climate variability has been a major driver in the long-term expansion of human activity. Historically, population growth, migration, and the development of technology have been, at least partly, in response to the driver of climate variability and its influence on the complex web of human activities. However, as modern industrial society becomes more sophisticated and integrated, future climate variability will likely have a more profound influence than has been the case in the past. For example, if a period similar to the 'little ice age' of 1645-1715 [1] were to recur in western society, the resulting change in energy demand would have major consequences for energy prices and possibly economic stability. Aside from such relatively recent and geographically localized events, there is of course significant long-term variability in the Earth's climate. The advance and retreat of ice sheets appears to be forced by the Milankovitch cycles, which can trip the climate into periodic ice ages through stochastic resonance [2].

Aside from natural climate variability, anthropogenic climate change has become a major issue due to the industrial release of carbon dioxide and other emissions. There is strong empirical evidence that such emissions are leading to an enhanced greenhouse effect and a rise in mean global temperatures. While natural climate variability has been of a greater magnitude, it is the apparent rapid change in the global mean temperature that raises concerns. It is assumed that a doubling of carbon 
dioxide concentration from a pre-industrial value of 280-560 ppm will lead to an increase in mean global temperature of order $1.75 \mathrm{~K}$ [3], although some studies indicate more significant warming [4]. Proxy records appear to show that the mean global temperature has already risen by $0.6 \pm 0.2 \mathrm{~K}$ during the last century, although there is still debate as to the appropriate use of such data [5]. As will be seen in section 3.2, a doubling of carbon dioxide concentration is estimated to require a reduction in total solar insolation of order 1.8 per cent to mitigate its effects.

Most attention and effort has been focused on emission reductions in order to minimize the peak concentration of carbon dioxide later in this century. While such approaches have dominated the debate on anthropogenic climate change, there is a growing discussion of engineering solutions, which can be deployed in addition to emission reductions, or as an alternative method of mitigation should emission controls prove to be politically unobtainable. While even the discussion of such geoengineering solutions can be controversial, it seems prudent to investigate geoengineering both as an effective tool to mitigate the effects of short-term anthropogenic climate change, and perhaps more importantly, as a tool to deal with the extremes of long-term natural climate variability [6-8]. It should be noted that given the global effects of geoengineering, serious political issues will of course arise concerning any future deployment.

Last, it should be noted that simple climate models can possess multiple, overlapping equilibria with the same forcing from solar insolation [9]. These models help explain the fact that the climate can switch rapidly between different states and is sensitive to relatively small changes in solar insolation driven, for example, by the Milankovitch cycles. The intriguing possibility then arises that the complexity of the climate can be actively exploited, so that geoengineering can be deployed in a more subtle and sophisticated manner than has been considered in the past. By exploiting the non-linearity of the climate, it may be that geoengineering schemes can be considered which can actively control such transitions between climate states using only modest, localized engineering intervention. This is an exciting possibility for the future, which may significantly reduce the scale of endeavour required compared to the macro-engineering schemes discussed here.

\section{GEOENGINEERING}

\subsection{Climate response}

The definition of geoengineering is open to interpretation, since it can be argued that the industrial release of carbon dioxide and other emissions is a form of geoengineering, given the likely climatic change that it will bring about. However, geoengineering will be used here as a term to describe deliberate, active intervention to modify the climate in a controlled manner, with presumed beneficial effect. It will be assumed that space-based geoengineering schemes can be slowly deployed over a period as long as 100 years, either to directly mitigate the effects on the climate of an unconstrained rise in carbon dioxide emissions or to spread terrestrial mitigation costs by allowing a gradual conversion to low carbon technologies.

While geoengineering has a long history in various guises [8], it is only relatively recently that spacebased geoengineering using orbiting solar reflectors has been considered (although as early as 1929 Oberth discusses the use of reflectors for localized climate engineering [10]). While only large-scale ventures are investigated here, there are a range of other measures available including active carbon capture and sequestration from fossil fuels, natural carbon sequestration through ocean fertilization, cloud formation, modification of surface albedo through ice cover, and land use and the control of atmospheric emissivity through long-lived radiatively active gases (including carbon dioxide) [8].

Critics of geoengineering have argued that a coarse modification of total solar insolation will lead to a range of undesirable consequences. In particular, it has been argued that while carbon dioxide traps heat during both the day and night cycles, a reduction in solar insolation will only be experienced during the day cycle. In addition, the averaged effect of such geoengineering would be most pronounced at the equator, leading to a less distinctive diurnal cycle and lower temperature gradients with latitude.

The response of the terrestrial climate to a 1.8 per cent reduction in solar insolation and a doubling of carbon dioxide concentration has been investigated using a full complexity atmosphere model $[3,11]$. Contrary to expectations, simulation shows that reducing the total solar insolation will indeed largely compensate for increased atmospheric carbon dioxide, significantly ameliorating increases in both mean global temperatures and indeed local variations. Other secondary effects of anthropogenic climate change, such as major disruption to ocean currents due to an increase in fresh water flows to arctic seas are also apparently avoided. More recent analysis using a fully coupled ocean-atmosphere model confirms that a coarse reduction in solar insolation can control global mean temperature, although decreases in precipitation are found [12]. While these results are encouraging, it is clear that much more detailed investigation is required to determine the potential long-term effects of geoengineering. In addition, it is noted that space-based geoengineering does not deal with the issue of ocean acidification due to increasing carbon dioxide. 


\subsection{Terrestrial geoengineering schemes}

Most proposals for large-scale geoengineering involve modifying total solar insolation through scattering sunlight back to space. A range of strategies have been proposed to mitigate anthropocentric climate change, including large-scale deposition of scattering aerosols in the stratosphere on a global scale, either sulphur dioxide particles $[\mathbf{8}, \mathbf{1 3}]$ or di-electric aerosols $[14,15]$. Early proposals by Budyko (as described by Keith [7] and Schneider $[8]$ ) estimated that approximately $10^{7}$ tonnes of sulphur dioxide per annum are required to offset a doubling of carbon dioxide concentration, while Teller et al. $[14,15]$ estimated that approximately $10^{7}$ tonnes per annum of $\sim 100 \mathrm{~nm}$ aerosols are required to increase the Earth's albedo by 1 per cent. Teller et al. also demonstrated that by using optically resonant scattering particles the mass requirements for such a venture could be reduced to $10^{5}-10^{6}$ tonnes per annum, with either mesh microstructures or vast numbers of small 4-mm helium-filled aluminium balloons sized to float to $25 \mathrm{~km}$, with a long life in the stratosphere, but oxidizing rapidly in the troposphere. Recent estimates by Crutzen identify a requirement for $1-2 \times 10^{6}$ tonnes of sulphur per annum deposited in the stratosphere from balloons in the tropics [13].

Climate cooling following major volcanic eruptions, such as Mount Pinatubo in 1991, demonstrates that such active control is possible, although engineered ventures would of course be significantly better optimized. The Pinatubo eruption deposited an estimated $1.7 \times 10^{7}$ tonnes of sulphur dioxide into the atmosphere, leading to a mean hemispheric surface cooling of order $0.5 \mathrm{~K}$ [16]. The climate cooling from the Pinatubo eruption is likely to have been greater than the effective warming from anthropogenic carbon dioxide during 1991-1993. However, there are concerns that large-scale deposition of engineered aerosols may lead to changes in atmospheric chemistry, in particular enhancing ozone depletion and the production of acid rain from sulphates.

Other promising, near-term geoengineering schemes include the exploitation of the Twomey effect, proposed by Latham and Salter to increase the reflectivity of low-level ocean clouds [17]. Automated ocean vessels would spray fine salt aerosols to generate nucleation centres for cloud formation. A combination of the motion of the vessels and atmospheric circulation would ensure good spatial coverage. A fleet of some 1500 wind-driven vessels is estimated to be required with each vessel mass of order 300 tonnes, yielding a total system mass of order $5 \times 10^{5}$ tonnes.

\subsection{Space-based geoengineering schemes}

Aside from aerosol deposition (albedo modification) and carbon sequestration (atmospheric emissivity modification), large-scale geoengineering using orbiting reflectors has been considered by various authors to manipulate the total solar insolation [18-24]. These concepts centre on fabricating and deploying a large occulting disc (or many smaller discs) to reduce the total solar insolation in order to mitigate increased carbon dioxide emissions. For example, the use of vast numbers $\left(\sim 5 \times 10^{4}\right)$ of $100 \mathrm{~km}^{2}$, actively controlled occulting discs in the Earth's orbit has been considered, but would likely lead to an apparent flickering of the Sun ( $\sim 2$ per cent amplitude) and would create a significant orbital debris hazard [7]. In addition, various proposals for an artificial ring of passive scattering particles in the Earth's orbit have been documented with a mass of order $2 \times 10^{9}$ tonnes $[\mathbf{2 1}, \mathbf{2 5}]$. A recent addition to this class of concepts is the use of clouds of dust grains located at the stable Earth-Moon triangular Lagrange points $L_{4}$ and $L_{5}$ [26]. In this scheme of order $2 \times 10^{11}$ tonnes of lunar or cometary dust is deposited in the EarthMoon system forming clouds at $L_{4}$ and $L_{5}$. As with other occulting schemes, the dust would lead to a reduction in solar insolation to offset radiative forcing by carbon dioxide. However, since each cloud only reduces solar insolation for a relatively short period each month, when the cloud is between the Earth and the Sun, significant mass is required to ensure a large optical depth and so useful cooling on average.

A perhaps more effective scheme is to station a large occulting disc (or discs), typically with a total mass of order $10^{7}-10^{8}$ tonnes, close to the Sun-Earth $L_{1}$ (Lagrange) equilibrium point some $1.5 \times 10^{6} \mathrm{~km}$ sunwards of the Earth. The equilibrium location at the $L_{1}$ point is unstable, necessitating the use of active control using solar radiation pressure. Indeed, the use of a large numbers of smaller occulting discs proposed by McInnes would mitigate the potentially catastrophic effect of the loss a single large disc [23].

The use of $L_{1}$ for geoengineering has been recently revisited by Angel who proposes swarms of engineered thin film refractive (rather than reflective) discs with a total mass of order $2 \times 10^{7}$ tonnes [24]. Rather than directly reflect solar radiation, the refracting discs scatter sunlight to avoid the Earth's disc, but are highly engineered thin film devices that require terrestrial fabrication and launch at extremely high cost. However, it is possible that much simpler partly reflecting discs could be fabricated in-situ from near-Earth asteroid resources [23]. There is a trade-off between the total mass of a geoengineering scheme and the engineering challenge of fabrication and deployment, as will be discussed later in section 5 .

As has been noted elsewhere [27], a key advantage of using large solar reflectors for geoengineering is the vast energy leverage obtained in a relatively short time. The total accumulated solar energy intercepted by the reflector quickly grows beyond the energy required for its fabrication, leading to a highly efficient tool for climate engineering. While solar reflectors offer many 
advantages, there are clearly challenges associated with the fabrication and active control of such large, gossamer structures. Again, it is almost certain that such structures would be fabricated in-orbit, either using lunar material or material processed from a suitable near-Earth asteroid. Therefore, a prerequisite for space-based geoengineering is the capability to effectively and economically exploit the resources of the moon or asteroids. The issues associated with the fabrication of large solar reflectors will be discussed later in section 5 .

\section{CLIMATE DYNAMICS}

\subsection{Energy balance model}

In order to determine the requirements for geoengineering, it is necessary to investigate the response of the Earth's climate to large-scale engineering intervention. In particular, the response of the global mean temperature to changes in total solar insolation is of interest, as are any non-linear effects that would pose a risk of unintended and potentially catastrophic modifications to the climate. By determining an approximate relationship between global mean temperature and solar insolation, the requirements for space-based geoengineering can be estimated and the scale of the venture can be determined. The energy balance model (EBM) is an extremely simple climate model that captures the essential large-scale features of the Earth's climate dynamics [28]. This low-order (so-called zero-dimensional) model clearly does not contain the sophistication of numerical general circulation models, but does allow insights into the underlying processes at work. The EBM assumes that any change to the heat balance of the Earth is simply due to an inequality between absorbed heat flux $Q_{\text {IN }}$ and emitted heat flux $Q_{\text {OuT. }}$. Therefore, assuming some mean specific heat capacity $C$ per unit area and global mean temperature $T$, the dynamics of the Earth's climate can be written simply as

$$
C \frac{\mathrm{d} T}{\mathrm{~d} t}=Q_{\mathrm{IN}}-Q_{\text {OUT }}
$$

so that

$$
C \frac{\mathrm{d} T}{\mathrm{~d} t}=Q(1-\alpha)-\varepsilon \sigma T^{4}
$$

where $Q\left(342.5 \mathrm{~W} / \mathrm{m}^{2}\right)$ is the total solar insolation, $\alpha(\sim 0.3)$ is the mean planetary albedo, $\varepsilon$ is the mean atmospheric emissivity $(\sim 0.62)$, and $\sigma(5.67 \times$ $\left.10^{-8} \mathrm{~W} / \mathrm{m}^{2} / \mathrm{K}^{4}\right)$ is the Stefan-Bolztman constant [28]. It should be noted that the solar insolation is defined in terms of the solar constant $F\left(1370 \mathrm{~W} / \mathrm{m}^{2}\right)$ as $Q=F / 4$, since the Earth presents only a circular cross-sectional area to the incoming flux but radiates over its entire spherical surface area. Assuming that the climate is in equilibrium $(\mathrm{d} T / \mathrm{d} t=0)$, a naive initial estimate of the Earth's mean temperature can be obtain from equation (1b) as

$$
\bar{T}=\left[\frac{Q(1-\alpha)}{\varepsilon \sigma}\right]^{1 / 4}
$$

which yields an estimated mean temperature of $14.0^{\circ} \mathrm{C}(287 \mathrm{~K})$, in good agreement with the observed global mean temperature. In order to provide a more accurate EBM, an empirical relationship that models the emitted heat flux $Q_{\text {out }}$ can be used. This more complete EBM can then be written as

$$
C \frac{\mathrm{d} T}{\mathrm{~d} t}=Q[1-\alpha(T)]-A-B T
$$

where the coefficients for $Q_{\text {out }}$ are determined from empirical observational data as $A=204 \mathrm{~W} / \mathrm{m}^{2}$ and $B=2.17 \mathrm{~W} / \mathrm{m}^{2} / \mathrm{K}[\mathbf{2 8}]$. Again, assuming that the climate is in equilibrium $(\mathrm{d} T / \mathrm{d} t=0)$, a new estimate of the Earth's mean temperature can be found from equation (3) as

$$
\bar{T}=\frac{Q(1-\alpha)-A}{B}
$$

This new estimate of the global mean temperature is found to be $16.5^{\circ} \mathrm{C}(289.5 \mathrm{~K})$, which is again in good agreement with the observed global mean temperature. Again, the EBM is used here solely to provide an estimate of the scale of geoengineering required.

\subsection{Climate cooling}

As discussed in section 1 , it is generally agreed that there has been an increase in the global mean temperature of order $0.6 \pm 0.2 \mathrm{~K}$ during the last century. This rise in global mean temperature is specifically due to a significant increase in radiatively active gases in the atmosphere, principally carbon dioxide, leading to a reduction in the effective emissivity of the atmosphere. The reduction in emissivity can be expressed as a change in radiative forcing. For a doubling of the carbon dioxide content in the atmosphere it can be shown that the coefficient $A$ is reduced by $4.17 \mathrm{~W} / \mathrm{m}^{2}$, resulting in lower re-radiation of heat due to an enhanced greenhouse effect [3]. It is noted that more recent estimates of forcing are of order $3.7 \mathrm{~W} / \mathrm{m}^{2}$. However, Angel [26] also uses the data from reference [3] and so the same forcing is used here to allow for direct comparison. In principle, this change in radiative forcing can be offset by a reduction in the effective solar insolation through geoengineering. From equation (4), the required change in solar insolation $\delta Q$ to offset a change in radiative forcing $\delta A$ while maintaining the same mean global temperature is given by

$$
\delta Q=Q-\frac{B \bar{T}+(A-\delta A)}{1-\alpha}
$$


Therefore, using equation (5) the simple EBM shows that to maintain the mean temperature of $17^{\circ} \mathrm{C}(290 \mathrm{~K})$ in the presence of a doubling of the carbon dioxide content of the Earth's atmosphere, a reduction in solar insolation $\delta Q / Q$ of 1.7 per cent is required. The requirements to engineer such a reduction in effective solar insolation will be determined later in section 4 . Again, it is noted that numerical studies of the effect of geoengineering have demonstrated that reducing solar insolation appears to compensate for increased atmospheric carbon dioxide, significantly ameliorating increases in global mean temperatures $[\mathbf{3}, \mathbf{1 1}, \mathbf{1 2}]$.

Assuming that such a reduction in solar insolation $\delta Q$ can be engineered, the response of the mean global temperature can be obtained from equation (3). If the change in insolation is small, so that the albedo is fixed, there is a change in mean global temperature such that

$$
T(t)=\bar{T}+\frac{\delta Q}{B}(1-\alpha)\left[1-\exp \left(\frac{-t}{\tau}\right)\right]
$$

where $\tau=C / B$ is the relaxation time of the system. By considering the heat capacity of the top $70 \mathrm{~m}$ of the oceans (mixing layer), it is found that $\tau \sim 3$ years, so that the response of the climate to changes in insolation is slow, but at an acceptable time scale for a geoengineering venture. For $t \gg \tau$ the resulting change in mean global temperature is of order $(1-\alpha) \delta Q / B$, so that a reduction in solar insolation $\delta Q / Q$ of 1.7 per cent to offset a doubling of the carbon dioxide content of the atmosphere is equivalent to reducing the mean global temperature by approximately $2 \mathrm{~K}$. The requirements to engineer such a reduction in solar insolation using a large occulting disc (or discs) will be considered in the next section.

\section{ENGINEERING REQUIREMENTS}

\subsection{Occulting solar discs}

The concept of using a large occulting disc (or discs) near the Sun-Earth $\mathrm{L}_{1}$ equilibrium point to reduce solar insolation has been discussed by various authors, as noted in section 2.3. In this section, it will be shown that there is in fact a minimum system mass which can be obtained if the disc is positioned at an optimum location along the Sun-Earth line, sunward of the classical $L_{1}$ Lagrange point. This optimum location is found from an analysis of the three-body mechanics of the problem with the addition of solar radiation pressure on the disc [29]. The location of the disc can be optimized since the solar radiation pressure exerted on the disc will generate a new equilibrium position, sunward of the classical $L_{1}$ Lagrange point. If the disc mass is reduced, the solar radiation pressure induced acceleration will increase and so the equilibrium point will be displaced sunward of the classical $L_{1}$ point [22]. However, as the disc is displaced sunward the required disc area to maintain the necessary reduction in solar insolation at the Earth will grow, leading to an increase in disc mass. These two processes must then be balanced in order to minimize the total disc mass through an optimum choice of disc location.

\subsection{Occulter orbit}

Now that the required change in solar insolation has been obtained from section 3.1, a suitable occulting disc (or discs) can be sized. For a disc of radius $R_{\mathrm{S}}$ at some distance $r_{\mathrm{S}}$ from the Earth, the disc will subtend a solid angle $\Omega_{\mathrm{S}}$ of $\pi R_{\mathrm{S}}^{2} / r_{\mathrm{S}}^{2}$, as shown in Fig. 1 . Similarly the Sun, of radius $R_{\mathrm{O}}$ at distance $r_{\mathrm{O}}$ from the Earth, will subtend a solid angle $\Omega_{\mathrm{O}}$ of $\pi R_{\mathrm{O}}^{2} / r_{\mathrm{O}}^{2}$, so that the disc will partially occult the Sun and reduce the insolation at the Earth by a factor $\Omega_{\mathrm{S}} / \Omega_{\mathrm{O}}$. It should be noted that by partly occulting the solar disc the solar flux $F$ is reduced such that $\delta Q=\delta F / 4$; however, the relative change in insolation $\delta Q / Q$ is identical to the relative change in flux $\delta F / F$. Therefore, the reduction in insolation produced by the occulting disc is defined by

$$
\frac{\delta Q}{Q}=\left(\frac{R_{\mathrm{S}}}{R_{\mathrm{O}}}\right)^{2}\left(\frac{r_{\mathrm{O}}}{r_{\mathrm{S}}}\right)^{2}
$$

Using equation (7), the required disc radius may now be obtained as a function of its distance $r_{\mathrm{S}}$ from the Earth to provide the required change in solar insolation of $\delta Q / Q$ as

$$
R_{\mathrm{S}}=R_{\mathrm{O}}\left(\frac{r_{\mathrm{S}}}{r_{\mathrm{O}}}\right)\left(\frac{\delta Q}{Q}\right)^{1 / 2}
$$

However, before the occulting disc is sized, the optimum distance of the disc from the Earth can be determined to minimize the total disc mass. As discussed in section 2.3 , the disc will be located near the

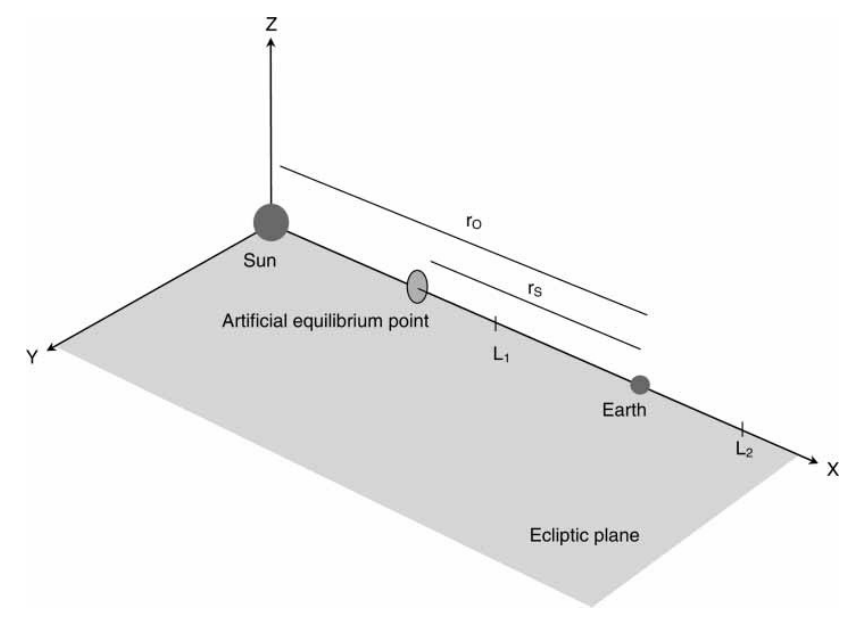

Fig. 1 Occulting solar disc stationed along the SunEarth line at an artificial equilibrium point. $L_{1}$ and $L_{2}$ are the classical three-body equilibrium points 
$L_{1}$ Sun-Earth equilibrium point. However, due to the solar radiation pressure acting on the disc, the equilibrium point will be displaced sunward of the classical $L_{1}$ point. A trade-off therefore exists between lowering the disc mass and displacing the equilibrium point sunward, and ultimately increasing the disc mass due to the increased disc area to provide the required partial occultation of the Sun. This trade-off leads to an optimum disc location that will minimize the total mass of the system.

The condition for equilibrium of the occulting disc in the Sun-Earth three-body problem can be determined from a simple force balance. The eccentricity of the Earth's orbit is neglected as is the lunar gravitational perturbation. Although the general solution for artificial equilibria for a reflector is known [29], only a simple one-dimensional problem need be considered here to locate the displaced equilibrium point. Similarly, it will be assumed that the disc station-keeps close to the Sun-Earth line to provide direct shadow. Since the mass of the Earth $M_{\mathrm{E}}$ is essentially negligible relative to the solar mass $M_{\mathrm{O}}$, the centre-of-mass of the Sun-Earth system will be taken as being located at the centre of mass of the Sun, as shown in Fig. 1. This approximation has a negligible effect on the subsequent analysis. The condition for equilibrium may now be obtained by balancing the gravitational force from the Sun and the Earth, the centripetal force and the solar radiation pressure induced acceleration experienced by the occulting disc $a_{\mathrm{S}}$ such that

$$
\begin{aligned}
& \frac{G M_{\mathrm{E}}}{r_{\mathrm{S}}^{2}}-\frac{G M_{\mathrm{O}}}{\left(r_{\mathrm{O}}-r_{\mathrm{S}}\right)^{2}}+\omega^{2}\left(r_{\mathrm{O}}-r_{\mathrm{S}}\right)+a_{\mathrm{S}}=0 \\
& \omega=\sqrt{\frac{G M_{\mathrm{O}}}{r_{\mathrm{O}}^{3}}}
\end{aligned}
$$

where $\omega$ is the orbital angular velocity of the Earth relative to the Sun and $G$ is the gravitational constant. The inverse square solar radiation pressure induced acceleration experienced by the occulting disc of mass $M_{\mathrm{S}}$ and area $A_{\mathrm{S}}$ may be written as

$$
a_{\mathrm{S}}=\frac{2 \kappa P_{\mathrm{E}} A_{\mathrm{S}}}{M_{\mathrm{S}}}\left(\frac{r_{\mathrm{O}}}{r_{\mathrm{O}}-r_{\mathrm{S}}}\right)^{2}
$$

where $P_{\mathrm{E}}\left(4.56 \times 10^{-6} \mathrm{~N} / \mathrm{m}^{2}\right)$ is the solar radiation pressure experienced by an absorbing surface at 1 astronomical unit $\left(r_{\mathrm{O}}\right)$ and $\kappa$ is a function of the optical properties of the disc. It can be shown [30] that for a specular reflector with Lambertian thermal re-emission the function $\kappa$ is given by

$$
\kappa=\frac{1}{2}\left[(1+\eta)+\frac{2}{3}(1-\eta) \frac{\varepsilon_{\mathrm{F}}-\varepsilon_{\mathrm{B}}}{\varepsilon_{\mathrm{F}}+\varepsilon_{\mathrm{B}}}\right]
$$

where $\eta$ is the specular reflectivity, $\varepsilon_{\mathrm{F}}$ is the emissivity of the front (Sun facing) side of the disc, and $\varepsilon_{\mathrm{B}}$ is the emissivity of the rear (Earth facing) side of the occulting disc, while the disc has an area $A_{\mathrm{S}}$ of $\pi R_{\mathrm{S}}^{2}$. Using equations (8) and (10), the disc mass $M_{\mathrm{S}}$ may now be written as

$$
M_{\mathrm{S}}\left(r_{\mathrm{S}}\right)=2 \pi \kappa P_{\mathrm{E}} R_{\mathrm{O}}^{2}\left(\frac{\delta Q}{Q}\right)\left(\frac{r_{\mathrm{S}}}{r_{\mathrm{O}}-r_{\mathrm{S}}}\right)^{2} \frac{1}{a_{\mathrm{S}}\left(r_{\mathrm{S}}\right)}
$$

where $a_{\mathrm{S}}$ is determined from equation (9). Since equation (12) is now a function of $r_{\mathrm{S}}$ only, the variation of the mass of the occulting disc with location along the Sun-Earth line can be investigated to attempt to minimize the disc mass.

\subsection{Occulter sizing}

The mass of the occulting disc may now be determined for a required reduction in solar insolation. For a fixed disc area, changing the disc mass will alter the solar radiation pressure acceleration experienced by the disc and so will influence the location of the equilibrium point. Assuming a reduction $\delta Q / Q$ of 1.7 per cent, as discussed in section 3.2, the variation of the disc mass with equilibrium location is shown in Fig. 2, where it can be seen that there are two limiting conditions. First, as the disc is located closer to the classical interior $L_{1}$ equilibrium point $1.50 \times 10^{6} \mathrm{~km}$ from the Earth, the disc mass grows and is unbounded as the classical Lagrange point is approached. This growth in mass is required to reduce the solar radiation pressure induced acceleration experienced by the disc, which would otherwise displace the equilibrium point sunward. Similarly, as the location of the disc is moved sunwards, the required mass of the disc will fall due to the increased solar radiation pressure

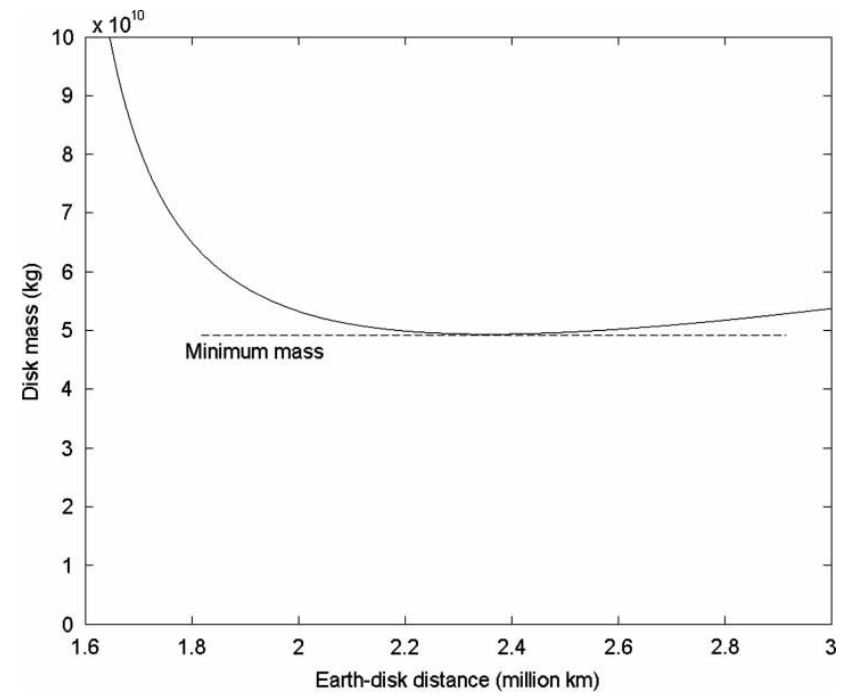

Fig. 2 Optimum occulting disc location $(\kappa=0.17)$. The classical $L_{1}$ point is at a distance of 1.5 million $\mathrm{km}$ from the Earth 
induced acceleration required for equilibrium. However, as the disc is moved sunwards significantly from the classical $L_{1}$ point, its mass will start to grow as the disc area increases to maintain the required solid angle subtended at the Earth to reduce the total solar insolation. These two opposing processes lead to a minimum disc mass, as can be seen in Fig. 2 .

The minimum disc mass can now be determined by finding the turning point of equation (12). It can be seen that there is a single location that will minimize the disc mass, independent of the required reduction in solar insolation or the disc optical properties. This location can be found by minimizing the function

$$
f\left(r_{\mathrm{S}}\right)=\left(\frac{r_{\mathrm{S}}}{r_{\mathrm{S}}-r_{\mathrm{O}}}\right)^{2} \frac{1}{a_{\mathrm{S}}\left(r_{\mathrm{S}}\right)}
$$

where it is found that $f^{\prime}\left(r_{\mathrm{S}}\right)=0$ when the disc location $r_{\mathrm{S}}$ is $2.36 \times 10^{6} \mathrm{~km}$ from the Earth. This optimum location is sunward of the classical interior Lagrange point at $1.50 \times 10^{6} \mathrm{~km}$ and again is independent of the disc properties, representing the true optimum location for an occulting disc (or discs). Assuming that the disc must provide a reduction in solar insolation $\delta Q / Q$ of 1.7 per cent, a disc with an effective radius of $1450 \mathrm{~km}$ (or an equivalent area from a large number of smaller discs) and a total mass of $2.6 \times 10^{8}$ tonne is required if $\kappa \sim 0.91$, representative of a reflecting metallic occulting disc.

However, if $\eta \sim 0$ and $\varepsilon_{\mathrm{F}} \sim 0$ then $\kappa \sim 0.17$, representative of a non-reflecting black occulting disc, resulting in substantial mass savings, although the optimum location of the reflector remains unchanged. In this case a total mass of $5.2 \times 10^{7}$ tonne is required, again with an effective radius of $1450 \mathrm{~km}$, as detailed in Tables 1 and 2. A non-reflecting disc could in principle be fabricated using a thin layer of carbon vacuum deposited on a metal film [30]. For in-situ manufacturing using a small near-Earth asteroid (discussed in section 5) carbon is readily available. For comparison, the masses of a range of terrestrial engineering ventures are listed in Table 3. It can be seen that the Chinese Three Gorges Dam requires approximately $6 \times 10^{7}$ tonne of concrete, and so forms a structure with a comparable mass to the occulting disc (or discs). While the challenges posed by space-based geoengineering are clearly significant, it interesting to note that measured in terms of mass, such large-scale geoengineering represents a venture of comparable scale to current large-scale terrestrial engineering ventures.

Table 1 Occulting disc optical properties

\begin{tabular}{lllll}
\hline Disc type & $\eta$ & $\varepsilon_{\mathrm{F}}$ & $\varepsilon_{\mathrm{B}}$ & $\kappa$ \\
\hline A (reflecting) & 0.82 & 0.06 & 0.06 & 0.91 \\
B (non-reflecting) & 0 & 0.01 & 0.5 & 0.17 \\
\hline
\end{tabular}

Table 2 System level trade-off

\begin{tabular}{llll}
\hline & $\begin{array}{l}\text { Mass } \\
\text { (tonne) }\end{array}$ & Area $\left(\mathrm{km}^{2}\right)$ & $\begin{array}{l}\text { Areal } \\
\text { density } \\
\left(\mathrm{g} / \mathrm{m}^{2}\right)\end{array}$ \\
\hline $\begin{array}{l}\text { Geoengineering concept } \\
\text { cloud [26]) }\end{array}$ & $2.1 \times 10^{11}$ & - & - \\
$\begin{array}{c}\text { Pearson (Earth orbit dust } \\
\text { ring [25]) }\end{array}$ & $2.3 \times 10^{9}$ & $1.1 \times 10^{8}$ & - \\
$\begin{array}{c}\text { McInnes A (Solar } L_{1} \\
\text { reflecting discs [22, 23]) }\end{array}$ & $2.6 \times 10^{8}$ & $6.57 \times 10^{6}$ & 40.2 \\
$\begin{array}{c}\text { McInnes B (Solar } L_{1} \\
\text { absorbing discs [22, 23]) }\end{array}$ & $5.2 \times 10^{7}$ & $6.57 \times 10^{6}$ & 7.9 \\
$\begin{array}{c}\text { Angel (Solar } L_{1} \text { refracting } \\
\text { discs [24]) }\end{array}$ & $2.0 \times 10^{7}$ & $4.70 \times 10^{6}$ & 4.2 \\
\hline
\end{tabular}

Table 3 Mass comparison with terrestrial engineering ventures

\begin{tabular}{lll}
\hline $\begin{array}{l}\text { Mass } \\
\text { scale }\end{array}$ & Mass (tonne) & Engineering venture \\
\hline $10^{5}$ & $6.5 \times 10^{5}$ & 'Knock Nevis' oil tanker (fully laden) \\
$10^{6}$ & $6 \times 10^{6}$ & Great pyramid of Giza \\
$10^{7}$ & $6 \times 10^{7}$ & Concrete used for three Gorges dam \\
$10^{8}$ & $2 \times 10^{8}$ & Water stored in London's reservoirs \\
$10^{9}$ & $7 \times 10^{9}$ & World annual $\mathrm{CO}_{2}$ emissions \\
\hline
\end{tabular}

While the analysis above has considered a single occulting disc, a more likely scenario would be the use of a large number of smaller discs with the same total area as that required for a single large disc. Such a swarm of discs may either be independent freeflying elements, or could be used to assemble a large occulter, as shown in Fig. 3. As will be discussed in section 5 below, the mass requirements for an absorbing occulter $[\mathbf{2 2}, \mathbf{2 3}]$ (as opposed to highly engineered refractors [24]) necessitates the use of lunar, or more likely near-Earth asteroid material. A scenario can be envisaged whereby individual elements are fabricated

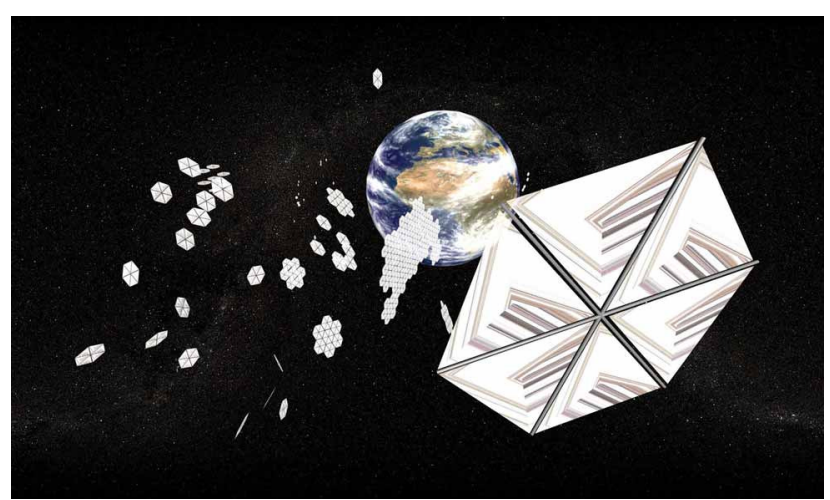

Fig. 3 Assembly of a large occulting disc from a swarm of discrete elements as a possible route to space-based geoengineering (Dario Izzo, $\mathrm{ESA} / \mathrm{ACT}$ ) 
from asteroid material and the total effective occulting area grows over a period of time (50-100 years), to match the required reduction in solar insolation to maintain a constant global mean temperature as carbon emissions rise. Such a gradual approach to geoengineering can also be used to reduce the peak costs of terrestrial carbon dioxide mitigation efforts.

\section{ENGINEERING CHALLENGES}

\subsection{Occulter fabrication}

The first steps towards fielding large orbiting solar reflectors are currently taking place through the accelerating development of solar sail propulsion for near-term space science missions [31]. A recent ground-based deployment test of a $20 \mathrm{~m} \times 20 \mathrm{~m}$ solar sail is shown in Fig. 4. Square solar sails with a side of order $100 \mathrm{~m}$ and an areal density of order $5 \mathrm{~g} / \mathrm{m}^{2}$ are foreseen, although concepts exist for both extremely large disc sails $(\sim 1000 \mathrm{~m})$ and low areal densities $\left(\sim 0.1 \mathrm{~g} / \mathrm{m}^{2}\right)$ using thin metallic film. These terrestrially fabricated reflectors can provide a route towards the fabrication of reflectors from in-situ resources such as near-Earth asteroids.

The use of occulting discs for geoengineering has been discussed in some detail in sections 3 and 4 , with a mass of order $5 \times 10^{7}$ tonne required to offset a doubling of atmospheric carbon dioxide. Clearly, the fabrication of a swarm of occulting discs with a mass of order $10^{7}$ tonne would require a capability to exploit in-situ resources such as near-Earth asteroids (although Angel envisages terrestrial fabrication of thin film refracting discs, and hence launch to $\left.\mathrm{L}_{1}[24]\right)$. For example, the mass requirements for the minimum mass occulting disc (or discs) can be satisfied by small M-type asteroids, which are abundant in nickel-iron materials and carbon [32]. A range of

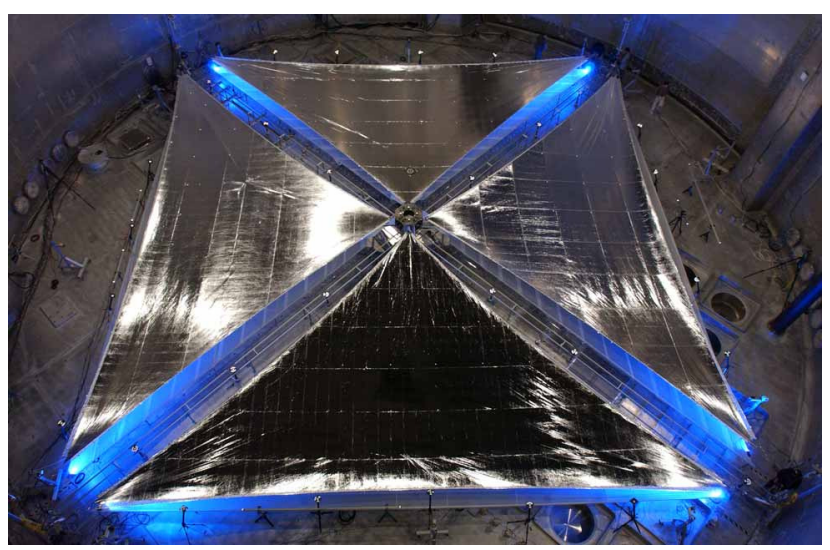

Fig. 4 Completed deployment test of a $20 \times 20 \mathrm{~m}$ solar sail to assess the use of storable booms and thin film reflectors (NASA) novel technologies are currently under investigation to deflect small near-Earth asteroids for hazard mitigation purposes [33-37]. However, the same technologies could, in principle, be used to capture a small near-Earth asteroid in the vicinity of the $L_{1}$ point for subsequent resource extraction, particularly for a small near-Earth asteroid in a low-energy orbit.

It will be assumed that the occulter is fabricated from thin metallic film (possibly with a carbon coating) processed from such a near-Earth asteroid, and that the asteroid has a bulk density half that of iron $\left(7860 \mathrm{~kg} / \mathrm{m}^{3}\right)$ to account for the non-metal content. It is then found that a small M-type asteroid with a radius of only $145 \mathrm{~m}$ will provide the required mass for fabrication of the occulting disc (or discs). The asteroid would require to be processed in-situ, probably using solar heating, and the metallic products extruded into thin film for fabrication of the discs. If it is assumed that a modest sized terrestrially fabricated solar reflector is initially deployed, then the time required to process the asteroid can be estimated. For example, a $500 \mathrm{~m}$ radius disc reflector will intercept a solar flux equivalent to approximately $1 \mathrm{GW}$ of power at 1 astronomical unit. If the disc has an areal density of order $1 \mathrm{~g} / \mathrm{m}^{2}$, its total mass is of order $800 \mathrm{~kg}$, well within the Earth escape capacity of a large commercial launch vehicle. Assuming the asteroid material is liberated by focusing this energy to raise the local surface temperature above the melting point of the metallic component (heat of fusion of iron $\sim 2.8 \times 10^{5} \mathrm{~J} / \mathrm{kg}$ ), the maximum rate of production of mass is potentially up to $3.6 \times 10^{3} \mathrm{~kg} / \mathrm{s}$ resulting in complete processing of the asteroid on a timescale of order 150 days.

Clearly, there are significant engineering challenges associated with extruding the liberated asteroid material into thin film, although in principle, thin film fabrication could be automated using vapour deposition. The hard vacuum required to allow vapour deposition is already provided by the space environment with the metal vapour condensed onto a cold substrate and separated after rapid cooling. As early as the late 1960s terrestrial laboratory experiments were performed to manufacture thin metal films for solar sails [38]. These initial studies used either a rotating, rigid cylindrical substrate or a flexible rotating band. By altering the speed of rotation of the substrate device, and by using masks, the thickness of the metal film could be varied to provide, e.g. additional strength at the edges of the film. Small-scale laboratory processes fabricated coarse films with a thickness of order $10 \mu \mathrm{m}$. Scaling such a process to fabricate the required effective area of occulting discs on a timescale of 50-100 years would clearly be a major challenge. For example, fabricating the required area of thin film in 100 years corresponds to a production rate of order $2000 \mathrm{~m}^{2} / \mathrm{s}$. If this is achieved using $10 \mathrm{~m}$ wide strips of film being separated from a cold roller a $1 \mathrm{~m} / \mathrm{s}$, some 200 vapour deposition 
units would be required. Clearly the deployed total mass of such a manufacturing system would be large, but would still be insignificant relative to the mass requirements for direct launch of the swarm of discs from the Earth. Such a gradual approach to geoengineering over a 50-100 year timescale can be used to reduce the peak costs of terrestrial carbon dioxide mitigation costs, in addition to direct compensation for increasing atmospheric carbon dioxide.

\subsection{Systems level trade-off}

The use of space-based geoengineering has been reviewed and a concept for occulting discs presented with a total deployed mass of $5.2 \times 10^{7}$ tonnes [22, 23]. As can be seen from Table 2, this is somewhat more than the scheme proposed by Angel $\left(2 \times 10^{7}\right.$ tonnes $)$ using terrestrially manufactured refracting discs [24], but significantly less than the dust cloud schemes proposed by Pearson $\left(2 \times 10^{9}\right.$ tonne $)$ [25] and Struck $\left(2 \times 10^{11}\right.$ tonne $)[\mathbf{2 6}]$.

Angel proposes a swarm of engineered refractive (rather than reflective) discs with a total mass of order $2 \times 10^{7}$ tonnes [24]. The refracting discs are highly engineered thin film devices that require terrestrial fabrication and launch at extremely high cost. McInnes proposes simple occulting discs [22, 23] with a total mass of order $5 \times 10^{7}$ tonnes that could be fabricated in-situ from near-Earth asteroid resources. Pearson and Struck propose clouds of coarse dust grains with a mass of $2 \times 10^{9}-10^{11}$ tonnes, which do not require any form of processing $[\mathbf{2 5}, \mathbf{2 6}$. There is a therefore a trade-off between the total mass of a geoengineering scheme and the engineering challenge and cost of fabrication and deployment. Angel [24] estimates a cost of $\$ 50$ per kg to manufacture highly engineered thin film refracting discs with a further $\$ 50$ per $\mathrm{kg}$ for launch to $\mathrm{L}_{1}$. By using in-situ resources launch costs are essentially eliminated, while the use of simple occulting discs or un-processed dust [26] would greatly reduce the specific cost (cost per unit mass), even although the deployed mass may be greater than for engineered refracting discs. Once available, the use of in-situ resources is therefore likely to substantially reduce the cost of space-based geoengineering significantly bellow the $\$ 100$ billion per year estimate of Angel. Such systems level issues must be addressed in future evaluation of competing geoengineering concepts.

Last, while the costs of such ventures appear daunting at present, a 100 year programme would benefit from accumulated world economic growth. For example, at the historical average growth rate of world gross domestic product of 4 per cent per annum, the world economy doubles in size every 17 years. A 100 year venture would see a world economy more than 50 times larger at the end of the project than the start.

\section{CONCLUSIONS}

The aim of this article has been to provide some insights into the possibilities offered by space-based geoengineering using orbiting solar reflectors. While such large-scale macro-engineering clearly requires a leap of the imagination over current large-scale terrestrial engineering, the natural and human-driven variability of the Earth's climate will necessitate some form of manipulation of the climate in the long term. Experience obtained from geoengineering may then pave the way towards engineering the climates of other planets, in particular Mars, through so-called terraforming. Again, while the scale of engineering discussed in this article may be daunting, the availability of vast quantities of freely available solar energy in space, and the active control of such energy using orbiting thin film solar reflectors may allow the possibility of large scale manipulation of planetary climates. Whether such possibilities are exploited in the future, both to mitigate natural and anthropogenic climate change on Earth and to unlock the resources of space, remains to be seen.

(C) Authors 2010

\section{REFERENCES}

1 Free, M. and Robock, A. Global warming in the context of the little ice age. J. Geophys. Res., 1999, 104, 19057-19070.

2 Muller, R. A. and MacDonald, G. J. Glacial cycles and astronomical forcing. Science, 1997, 277, 215-218.

3 Govindasamy, B. and Caldeira, K. Geoengineering Earth's radiation balance to mitigate $\mathrm{CO}_{2}$ induced climate change. Geophys. Res. Lett., 2000, 27, 2141-2144.

4 Stainforth, D. A., Aina, T., Christensen, C., Collins, M., Faull, N., Frame, D. J., Kettleborough, J. A., Knight, S., Martin, A., Murphy, J. M., Piani, C., Sexton, D., Smith, L. A., Spicer, R. A., Thorpe, A. J., and Allen, M. R. Uncertainty in predictions of the climate response to rising levels of greenhouse gases. Nature, 2005, 433, 403-406.

5 Mann, M. E., Bradley, R. S., and Hughes, M. K. Globalscale temperature patterns and climate forcing over the past six centuries. Nature, 1998, 392, 779-787.

6 Cicerone, R. J., Elliott, S., and Turco, R. P. Global environmental engineering. Nature, 1992, 356, 9.

7 Keith, D. W. Geoengineering the climate: history and prospect. Annu. Rev. Energy Environ., 2000, 25, 245-284.

8 Schneider, S. H. Earth systems engineering and management. Nature, 2001, 409, 417-421.

9 Emanuel, K. A simple model of multiple climate regimes. J. Geophys. Res., 2002, 107, ACL 4-1. DOI: 10.1029/2001DJ001002.

10 Oberth, H. Ways to spaceflight, NASA Technical Translation, TT F-622, 1972.

11 Govindasamy, B., Thompson, S., Duffy, P., Caldeira, K., and Delire, C. Impact of geoengineering schemes on the 
terrestrial biosphere. Geophys. Res. Lett., 2002, 29, 18.118.4. DOI: 10.1029/2002GL015911.

12 Lunt, D. J., Ridgwell, A., Valdes, P. J., and Seale, A. Sunshade world: a fully coupled GCM evaluation of the climatic impacts of geoengineering. Geophys. Res. Lett., 2008, 35, L12710.

13 Crutzen, P. J. Albedo enhancement by stratospheric sulfur injections: a contribution to resolve a policy dilemma? Clim. Change, 2006, 77, 211-219.

14 Teller, E., Wood, L., and Hyde, R. Global warming and ice ages: I. prospects for physics based modulation of global change, UCRL-231636/UCRL JC 128715, Lawrence Livermore National Laboratory, 1997.

15 Teller, E., Hyde, R., Ishikawa, M., Nuckolls, J., and Wood, L. Active climate stabilization: presently-feasible albedocontrol approaches to prevention of both types of climate change. In Proceedings of the Cambridge-MIT Institute Symposium on Macro-engineering options for climate change management and mitigation, Cambridge, 2004.

16 Hansen, J., Lacis, A., Ruedy, R., and Sato, M. Potential climate impact of Mount Pinatubo eruption. Geophys. Res. Lett., 1992, 19, 215-218.

17 Salter, S., Sortino, G., and Latham, J. Sea-going hardware for the cloud albedo method of reversing global warming. Philos. Trans. R. Soc. A., 2008, 366, 3989-4006.

18 Seifritz, W. Mirrors to halt global warming. Nature, 1989, 340, 603.

19 Early, J. T. Space-based solar shield to offset greenhouse effect. J. Br. Interplanet. Soc., 1989, 42, 567-569.

20 Hudson, H. A space parasol as a countermeasure against the greenhouse effect. J. Br. Interplanet. Soc., 1991, 44, 139-141.

21 Mautner, M. A space-based solar screen against climatic warming. J. Br. Interplanet. Soc., 1991, 44, 135-138.

22 McInnes, C. R. Minimum mass solar shield for terrestrial climate control. J. Br. Interplanet. Soc., 2002, 55, 307-311.

23 McInnes, C. R. Planetary macro-engineering using orbiting solar reflectors. In Macro-engineering: a challenge for the future, 2006, pp. 215-250 (Springer, Berlin).
24 Angel, R. Feasibility of cooling the Earth with a cloud of small spacecraft near the inner Lagrange point $\left(L_{1}\right)$. Proc. Natl. Acad. Sci., 2006, 103, 17184-17189.

25 Pearson, P., Oldson, J., and Levin, E. Earth rings for planetary environment control. Acta Astron., 2006, 58(1), 44-57.

26 Struck, C. The feasibility of shading the greenhouse with dust clouds at the stable lunar Lagrange points. J. Br. Int. Soc., 2007, 60, 82-89.

27 Mautner, M. and Parks, K. Space-based control of the climate. In Proceedings of the Space 90, 1990, pp. 11591169 (American Society of Civil Engineers).

28 McGuffie, K. and Henderson-Sellers, A. A climate modelling primer, 1997 (John Wiley, Chichester).

29 McInnes, C. R., McDonald, A. J. C., Simmons, J. F. L., and MacDonald, E. W. Solar sail parking in restricted threebody systems. J. Guid. Control Dyn., 1994, 17, 399-406.

30 Sanders, D. M., Boercker, D. B., and Falbella, S. Coating technology based on the vacuum arc - a review. IEE Trans. Plasma Sci., 1990, 18, 883-894.

31 McInnes, C. R. Solar sailing: technology, dynamics and mission applications, 1999 (Springer, London).

32 Gehrels, T. (Ed.). Asteroids, 1979 (University of Arizona Press, Tucson)

33 Melsoh, H. J. Solar asteroid diversion. Nature, 1993, 366, 21.

34 Lu, E. T. and Love, S. G. Gravitational tractor for towing asteroids. Nature, 2005, 438, 177-178.

35 McInnes, C. R. Deflection of near-Earth asteroids by kinetic energy impacts from retrograde orbits. Planet. Space Sci., 2004, 52, 587-590.

36 McInnes, C. R. Near Earth object orbit modification using gravitational coupling. J. Guid. Control Dyn., 2007, 30(3), 870-873.

37 Vasile, M. and Colombo, C. Optimal impact strategies for asteroid deflection. J. Guid. Control Dyn., 2008, 31, 858-872.

38 Lippman, M. E. In-space fabrication of thin-film structures, NASA CR-1969, February 1972. 